Original Research Paper

\title{
A Convergence Theorem for Bivariate Exponential Dispersion Models
}

\author{
${ }^{1}$ Lila Ricci and ${ }^{2}$ Gabriela Boggio \\ ${ }^{I}$ Centro Marplatense de Investigaciones Matemáticas, Universidad Nacional de Mar del Plata, Mar del Plata, Argentina \\ ${ }^{2}$ Escuela de Estadística, Universidad Nacional de Rosario, Rosario, Argentina
}

Article history

Received: 16-05-2019

Revised: 24-06-2019

Accepted: 23-07-2019

Corresponding Authors:

Lila Ricci

CEMIM, UNMdP, MdP,

Argentina

E-mail: lricci@mdp.edu.ar

\begin{abstract}
Multivariate exponential dispersion models (MEDMs) were defined in 2013 by Jørgensen and Martínez. A particular case of MEDM is the bivariate Gamma model; in this article we prove that, under certain conditions, this is a limit distribution for $M E D M$ generated by bivariate regularly varying measures, extending a previous result given by the aforementioned authors for the univariate case. As necessary tools for proving the main result, we use bivariate regularly varying functions and bivariate regularly varying measures; we also state a bivariate version of Tauberian Karamata's theorems and a particular Karamata representation of bivariate slowly varying functions.
\end{abstract}

Keywords: Multivariate Exponential Dispersion Model, Regular Variation Karamata Representation

\section{Introduction}

The aim of this article is to extend to bi-dimensional space the result about Tauber type convergence of exponential dispersion models $(E D M) \mathrm{s}$ to a Gamma model, that has been proved by Jørgensen et al. (1994).

It was Tweedie (1947) pointed out the main properties of EDMs; but his ideas remained unknown for decades. On the other hand, Nelder and Wedderburn (1972) introduced a new class of statistical models named Natural Exponential Family, just when computational tools were being developed in such a way that it became possible to perform the required computations. Their importance comes from the fact that they can represent the error distribution in Generalized Linear Models (GLMs).

Later on, Jørgensen (1987) rescued Tweedie's ideas and defined an extended family of distributions named Exponential Dispersion Model. He published systematic studies of mathematical properties of EDMs in 1986 and 1987. In his own words, the main raison d'être of EDMs is to broaden the field of GLMs introduced by Nelder and Wedderburn (1972) allowing the researchers to choose between infinite probability distributions the one that optimally represents their data.

Under certain conditions, it has been proved that some EDMs converge to the Gamma distribution. An essential tool in the study of these domains of attraction has been the theory of regularly varying functions, they arise when the mean parameter goes to zero or infinity, while the dispersion parameter remains constant or asymptotically constant. That is why this convergence has been called of regular variation type. Using these resources Jørgensen et al. (1994) proved an important theorem, making it possible to asses Gamma convergence of some EDMs under weaker conditions than those required for asymptotic convergence of variance functions.

On the other hand, Jørgensen (2013; Jørgensen and Martínez, 2013) developed a unified methodology to build Multivariate Exponential Dispersion Models (MEDMs) with fixed known marginals and a flexible correlation structure. Based on previous univariate studies about EDMs convergence (Jørgensen et al., 1994; 2009) they could conjecture that previous results might be extended to those $M E D M s$ that they had just defined. Moreover, $M E D M S$ are important for practitioners because they broaden the parametric distribution family covered by GLMs derived from Multivariate Natural Exponential Family.

In the next Section we give the definition of MEDMs; then we define bivariate regular variation and we state Karamata's theorems and we prove an asymptotic property of $M E D M s$, that is the main result of this article. Finally, some conclusions are developed. There are also three appendixes with some detailed calculations.

\section{Multivariate Exponential Dispersion Models}

A method to obtain $M E D M s$ has been presented by Jørgensen (2013; Jørgensen and Martínez, 2013). It is based on an extended convolution method that ensures a 
$k+k(k+1) / 2$ parameters distribution for $\mathrm{k}$-dimensional models, with marginal distributions that belong to the same family; these new MEDMs have a flexible covariance structure. In this section the construction of the bivariate EDM will be detailed. Consider the probability density function:

$$
f^{*}(z ; \boldsymbol{\theta}, \lambda)=c(z ; \lambda) \exp \left[\boldsymbol{z}^{T} \boldsymbol{\theta}-\lambda \kappa(\boldsymbol{\theta})\right], \boldsymbol{z} \in \mathbb{R}^{k}
$$

where, $\boldsymbol{\theta} \in \Theta \subseteq \mathbb{R}^{k}, c(\cdot ;)$ is a suitable function and $\lambda$ is the weight parameter. The cumulant generating function (CGF) is:

$$
\lambda \kappa(s ; \theta)=\lambda\{\kappa(\theta+s)-\kappa(\theta)\} \text { for } \boldsymbol{s} \in \Theta-\theta
$$

and it characterizes the distribution.

The bivariate case of these new $M E D M s$ is obtained as follows: let $\boldsymbol{Z}=\left(Z_{1}, Z_{2}\right)^{T}$ be expressed as:

$$
\boldsymbol{Z}=\left(U_{1}, U_{2}\right)^{T}+\left(U_{1}, 0\right)^{T}+\left(0, U_{2}\right)^{T}
$$

where, the three terms are independent with CGFs given by $\left(s_{1}, s_{2}\right)^{T} \rightarrow \lambda_{12} \kappa\left(s_{1}, s_{2} ; \boldsymbol{\theta}\right)$, $\left(s_{1}, 0\right) \rightarrow \lambda_{1} \kappa\left(s_{1}, 0 ; \boldsymbol{\theta}\right)$ and $\left(0, s_{2}\right) \rightarrow \lambda_{2} \kappa\left(0, s_{2} ; \boldsymbol{\theta}\right)$ respectively. $\left(s_{1}, s_{2}\right)\left(s_{1}, s_{2}\right)^{T} \rightarrow \lambda_{12} \kappa\left(s_{1}, s_{2} ; \theta_{1}, \theta_{2}\right)$ Adding these three expressions we obtain the bivariate CGF:

$$
K^{*}\left(s_{1}, s_{2} ; \boldsymbol{\theta}\right)=\lambda_{12} \kappa\left(s_{1}, s_{2} ; \boldsymbol{\theta}\right)+\lambda_{1} \kappa\left(s_{1}, 0 ; \boldsymbol{\theta}\right)+\lambda_{2} \kappa\left(0, s_{2} ; \boldsymbol{\theta}\right)
$$

that characterizes the model denoted by $E D^{*}(\boldsymbol{\theta}, \boldsymbol{\Lambda})$. Given the additive property of $E D^{*}$, it can be seen that the marginals are models of the same family with weights $\lambda_{i i}=\lambda_{12}+\lambda_{i}, i=1,2$. The mean vector is $E(\mathbf{Z})=\lambda \odot \mu=\lambda \odot \dot{\kappa}(\theta)$, being $\lambda=\left(\lambda_{11}, \lambda_{22}\right)^{T}, \quad \dot{\kappa}(\theta)_{\mathrm{a}}$ vector with elements $[\dot{\kappa}(\theta)]_{i}=\frac{\partial \kappa(\theta)}{\partial \theta_{i}}$ and $\odot$ denotes the Hadamard product. The covariance matrix is:

$$
\operatorname{Cov}(\boldsymbol{Z})=\boldsymbol{\Lambda} \odot V(\boldsymbol{\mu})
$$

where, $\boldsymbol{\Lambda}=\left[\begin{array}{ll}\lambda_{11} & \lambda_{12} \\ \lambda_{12} & \lambda_{22}\end{array}\right], \quad V(\boldsymbol{\mu})=\ddot{\kappa} \circ \dot{\kappa}^{-1}(\mu)$ is the unit variance matrix and $\ddot{\kappa}$ is a matrix with elements $[\ddot{\kappa}(\theta)]_{i j}=\frac{\partial^{2} \kappa(\theta)}{\partial \theta_{i} \partial \theta_{j}}$.

The reproductive $M E D M$ is defined by the scale transformation $\boldsymbol{Y}=\left(Z_{1} / \lambda_{11}, Z_{2} / \lambda_{22}\right)^{T}$, the random vector $\boldsymbol{Y}$ has mean $\mu=\dot{\kappa}(\theta)$ and covariance matrix:

$$
\operatorname{Cov}(\boldsymbol{Y})=\left[\begin{array}{cc}
\sigma_{11} \mu_{1}^{2} & \sigma_{12} \phi \mu_{1} \mu_{2} \\
\sigma_{12} \phi \mu_{1} \mu_{2} & \sigma_{22} \mu_{2}^{2}
\end{array}\right]
$$

where, $\sigma_{i j}$ are the components of the dispersion matrix:

$$
\Sigma=\left[\begin{array}{cc}
\frac{1}{\lambda_{11}} & \frac{\lambda_{12}}{\lambda_{11} \lambda_{22}} \\
\frac{\lambda_{12}}{\lambda_{11} \lambda_{22}} & \frac{1}{\lambda_{22}}
\end{array}\right] ;
$$

we will denote it by $\boldsymbol{Y} \sim E D(\boldsymbol{\mu}, \boldsymbol{\Sigma})$. One slight disadvantage of the method is that only positive correlations are obtained. The parameter domain that ensures that $K^{*}\left(s_{1}, s_{2} ; \theta\right)$ is a $C G F$ could be broaden in order to admit negative correlations, but this issue remains to be investigated. With this in mind, Cuenin et al. (2015) give a variables-incommon method for constructing multivariate distributions admitting negative correlations, but it is restricted to Tweedie models.

\section{A Particular Case: Bivariate Gamma}

While passing from uni to multivariate distributions there is more than one direction to choose. In words of Letac: "While the names of distributions in $\mathbb{R}$ are generally unambiguous, at the contrary in the jungle of distributions in $\mathbb{R}^{k}$ almost nothing is codified outside of the Wishart and Gaussian cases".

Let us consider Kibble and Moran bivariate Gamma distribution as given by Kotz et al. (2000) whose cumulant function is:

$$
\kappa\left(\theta_{1}, \theta_{2}\right)=-\log \left(\theta_{1} \theta_{2}-\rho\right), \rho>0
$$

with domain:

$$
\Theta=\left\{\left(\theta_{1}, \theta_{2}\right), \theta_{1}<0, \theta_{2}<0, \theta_{1} \theta_{2}-\rho>0\right\}
$$

The mean vector is:

$$
\begin{aligned}
& E(\boldsymbol{Z})=\lambda \odot \dot{\kappa}\left(\theta_{1}, \theta_{2}\right) \\
& =\frac{1}{\rho-\theta_{1} \theta_{2}}\left[\begin{array}{l}
\left(\lambda_{12}+\lambda_{1}\right) \theta_{2} \\
\left(\lambda_{12}+\lambda_{2}\right) \theta_{1}
\end{array}\right] \\
& =\left[\begin{array}{c}
\lambda_{11} \mu_{1} \\
\lambda_{22} \mu_{2}
\end{array}\right]
\end{aligned}
$$

and the covariance matrix is:

$$
\operatorname{Cov}(\boldsymbol{Z})=\Lambda \odot \ddot{\kappa}\left(\theta_{1}, \theta_{2}\right)=\left[\begin{array}{cc}
\lambda_{11} \mu_{1}^{2} & \lambda_{12} \phi \mu_{1} \mu_{2} \\
\lambda_{12} \phi \mu_{1} \mu_{2} & \lambda_{22} \mu_{2}^{2}
\end{array}\right],
$$


where, $\phi=1-\frac{1}{2 \rho \mu_{1} \mu_{2}}\left(\sqrt{1+4 \rho \mu_{1} \mu_{2}}-1\right)$ as is demonstrated in Appendix C (a detailed treatment can also be seen in Boggio's (2019)). The moment generating function $(M G F)$ of the reproductive model in terms of $\mu$ is then:

$$
\begin{aligned}
& M(\boldsymbol{s} ; \boldsymbol{\mu}, \Lambda)=\left(1-\mu_{1} \frac{s_{1}}{\lambda_{11}}-\mu_{2} \frac{s_{2}}{\lambda_{22}}+\mu_{1} \mu_{2}(1-\phi) \frac{s_{1} s_{2}}{\lambda_{11} \lambda_{22}}\right)^{-\lambda_{12}} \\
& \times\left(1-\mu_{1} \frac{s_{1}}{\lambda_{11}}\right)^{-\lambda_{1}}\left(1-\mu_{2} \frac{s_{2}}{\lambda_{22}}\right)^{-\lambda_{2}}
\end{aligned}
$$

Note that when $\phi=0$, meaning independence, (1) becomes:

$$
M(\boldsymbol{s} ; \boldsymbol{\mu}, \Lambda)=\left(1-\mu_{1} \frac{s_{1}}{\lambda_{11}}\right)^{-\lambda_{11}}\left(1-\mu_{2} \frac{s_{2}}{\lambda_{22}}\right)^{-\lambda_{22}}
$$

\section{Bivariate Regular Variation and Karamata}

\section{Theorems}

In this section we present some definitions and results that will be needed in the next section. They include bivariate regular variation, Karamata Tauberian theorems about Laplace transforms and Karamata representation.

\section{Bivariate Regular Variation}

Regular variation functions were defined by J. Karamata (Goldie et al., 1987; de Haan, 1975), they behave asymptotically as their Laplace transforms. The next definition was given by Omey and Willekens (1989), extending the concept of regular variation to $\mathbb{R}_{+}^{2}$.

Defnition 1. A measurable function $u: \mathbb{R}_{+}^{2} \rightarrow \mathbb{R}_{+}$is regularly varying at infinity with indexes $\alpha, \beta \in \mathbb{R} \cdot$ if $\forall x$, $y>0$ and $t>0$, the limit:

$$
\lim _{\min (t, s) \rightarrow \infty} \frac{u(t x, s y)}{u(t, s)}=x^{\alpha} y^{\beta}
$$

exists and is finite.

We will denote a regularly varying function at infinity with indexes $\alpha$ and $\beta$ by $u \in V R(\alpha, \beta)_{\infty}$; analogously $u(x, y)$ is regularly varying at 0 if $u\left(\frac{1}{x}, \frac{1}{y}\right)$ is regularly varying at infinity and we will denote it by $u \in$ $V R(\alpha, \beta)_{0}$. If $\alpha=\beta=0$ the function is said to be slowly varying at infinity (zero) and we will denote it by $L \in V L_{\infty(0)}$. The concept of regular variation can be extended to measures as follows.
Defnition 2: A measure $v$ on $\mathbb{R}_{+}^{2}$ is said to vary regularly at infinity or zero with indexes $\alpha, \beta \in \mathbb{R}$ if the distribution function $\bar{v}(x, y)=v\{(0, x] \times(0, y]\}$ does.

\section{Bivariate Karamata's Theorems}

Next we extend a theorem stated by Jørgensen et al. (1994), that relates regular variation of a measure with regular variation of its Laplace transform. Hereafter the notation " $f(x) \sim k g(x)$ when $x \rightarrow \infty$ " means that $\lim _{x \rightarrow \infty} \frac{f(x)}{g(x)}=k$.

\section{Theorem 1 (Bivariate Karamata Tauberian Theorem).}

Let $v$ be a measure on $\mathbb{R}_{+}^{2}$ with Laplace transform $\omega(.,$.$) , then:$

$$
\begin{array}{r}
\bar{v}(t, s) \sim \frac{1}{\Gamma(\alpha+1) \Gamma(\beta+1)} t^{\alpha} s^{\beta} L(t, s) \Leftrightarrow \\
\omega\left(\frac{1}{t}, \frac{1}{s}\right) \sim t^{\alpha} s^{\beta} L(t, s)
\end{array}
$$

when $\min (t, s) \rightarrow \infty, L \in V L_{\infty}, \alpha$ and $\beta$ being non negative numbers and $\bar{v}$ the function given in Definition 2.

Proposition 1. The statement on the left is equivalent to affirm that $\bar{v} \in V R(\alpha, \beta)_{\infty}$ as is proved in Appendix $A$.

Now let $v$ be $a$ measure of the form:

$$
v(d x, d y)=g(x, y) x^{\alpha-1} y^{\beta-1} d x d y,
$$

$g$ being analytic and zero at $(0,0)$, then $v \in V R(\alpha, \beta)_{0}$. Theorem 1 allows us to say that the MGF of the natural exponential family generated by such a measure takes the form:

$M_{v}\left(\theta_{1}, \theta_{2}\right)=\left(-\theta_{1}\right)^{-\alpha}\left(-\theta_{2}\right)^{-\beta} L\left(-\theta_{1},-\theta_{2}\right), \theta_{1}, \theta_{2}<0$,

where $L\left(-\theta_{1},-\theta_{2}\right) \in V L_{\infty}$

On the other hand, de Haan and Resnick (1987) proved an extension of Karamata representation to the multivariate regular variation case; we are interested in the particular case of bivariate slow variation. A slowly varying function $L: \mathbb{R}_{+}^{2} \rightarrow \mathbb{R}$ can be represented as:

$L(x, y)=d(x, y) \exp \left\{\int_{1}^{\|(x, y)\|} \frac{a(t, t)}{t} d t\right\}$

where, $\quad d: \mathbb{R}_{+}^{2} \rightarrow \mathbb{R} \quad$ and $\quad a: \mathbb{R}_{+}^{2} \rightarrow \mathbb{R} \quad$ such that $\lim _{t \rightarrow \infty}\left|d(t x, t y)-d_{0}\right|=0 \quad$ for $\quad$ some $\quad 0<d_{0}<\infty \quad$ and $\lim _{t \rightarrow \infty} a(t, t)=0$. 


\section{The Main Result}

In the univariate case several convergence theorems have been proved considering three types of convergence: central limit type, infinitely divisible type and regular variation type (Nielsen, 2000; Jørgensen, 1997: p149). Our main result concerns convergence of regular variation type, where the dispersion parameter remains constant while the mean tends to zero or infinity. In this section we extend a theorem that has been proved in the univariate context to the bivariate case. This theorem asses the asymptotic equivalence between some bivariate EDM and the bivariate Kibble and Moran's Gamma distribution, with weaker assumptions than previous theorems. Extension to $\mathbb{R}_{+}^{k}$ is straightforward.

We will introduce now some notation in order to simplify next developments. Let the bivariate $E D M(\boldsymbol{\mu}$, A) be generated by a measure $v$ with support $S \subseteq(0, \infty) \times$ $(0, \infty)$. If $v$ is regularly varying at zero or infinity with index $\alpha>0$, then given (3) its $C G F$ is:

$\kappa(\theta)=-\alpha \log \left(\theta_{1}\right)-\alpha \log \left(\theta_{2}\right)+l\left(-\theta_{1},-\theta_{2}\right)$,

where, $l(x, y)=\log L(x, y)$. The mean value vector takes the form:

$$
\boldsymbol{\mu}=\left[\begin{array}{c}
\tau_{1}(\theta) \\
\tau_{2}(\theta)
\end{array}\right]=\left[\begin{array}{c}
-\frac{\alpha}{\theta_{1}}-\frac{\partial l(-\theta)}{\partial \theta_{1}} \\
-\frac{\alpha}{\theta_{2}}-\frac{\partial l(-\theta)}{\partial \theta_{2}}
\end{array}\right]
$$

We define functions $\delta_{i}\left(\mu_{1}, \mu_{2}\right), i=1,2$ as:

$$
\left[\begin{array}{l}
\delta_{1}\left(\mu_{1}, \mu_{2}\right) \\
\delta_{2}\left(\mu_{1}, \mu_{2}\right)
\end{array}\right]=\left[\begin{array}{l}
\theta_{1} \\
\theta_{2}
\end{array}\right]=\tau^{-1}(\boldsymbol{\mu})
$$

Now:

$$
\boldsymbol{\mu}=\left[\begin{array}{l}
\mu_{1} \\
\mu_{2}
\end{array}\right]=\left[\begin{array}{c}
-\frac{\alpha}{\delta_{1}(\boldsymbol{\mu})}-\frac{\partial l\left(-\delta_{1}(\boldsymbol{\mu}),-\delta_{2}(\boldsymbol{\mu})\right)}{\partial \delta_{1}(\boldsymbol{\mu})} \\
-\frac{\alpha}{\delta_{2}(\boldsymbol{\mu})}-\frac{\partial l\left(-\delta_{1}(\boldsymbol{\mu}),-\delta_{2}(\boldsymbol{\mu})\right)}{\partial \delta_{2}(\boldsymbol{\mu})}
\end{array}\right],
$$

giving:

$$
\mu_{i}+\frac{\partial}{\partial \delta_{i}(\boldsymbol{\mu})} l\left(-\delta_{1}(\boldsymbol{\mu}),-\delta_{2}(\boldsymbol{\mu})\right)=-\frac{\alpha}{\delta_{i}(\boldsymbol{\mu})}, i=1,2
$$

and the vector $\tau^{-1}(\boldsymbol{\mu})$ can be expressed as:

$$
\tau^{-1}(\mu)=\left[\begin{array}{l}
\delta_{1}(\boldsymbol{\mu}) \\
\delta_{2}(\boldsymbol{\mu})
\end{array}\right]=\left[\begin{array}{l}
\frac{-\alpha}{\mu_{1}+\frac{\partial l\left(-\tau^{-1}(\boldsymbol{\mu})\right)}{\partial \delta_{1}(\boldsymbol{\mu})}} \\
\frac{-\alpha}{\mu_{2}+\frac{\partial l\left(-\tau^{-1}(\boldsymbol{\mu})\right)}{\partial \delta_{2}(\boldsymbol{\mu})}}
\end{array}\right] .
$$

Now the main theorem can be stated.

Theorem 2: Let $\boldsymbol{Y} \sim E D(\boldsymbol{\mu}, \Sigma)$ be a bivariate EDM generated by the measure $v$ with support $S \subseteq(0, \infty) \times(0, \infty)$. Suppose that $v$ is regularly varying at zero or infinity with the same index on both variables. Given (3) and if $l(x, y)$ satisfies:

$\lim _{c \rightarrow 0(\infty)} \frac{1}{c} \frac{\partial l\left(-\delta_{1}(c \mu),-\delta_{2}(c \mu)\right)}{\partial \delta_{i}(c \mu)}=0, i=1,2$

for all $\boldsymbol{\mu} \in \mathbb{R}_{+}^{2}$ and $\Sigma=\left[\begin{array}{cc}\frac{1}{\lambda_{11}} & \frac{\lambda_{12}}{\lambda_{11} \lambda_{22}} \\ \frac{\lambda_{12}}{\lambda_{11} \lambda_{22}} & \frac{1}{\lambda_{22}}\end{array}\right]$ with $\lambda_{11}>0, \lambda_{22}>0$ and

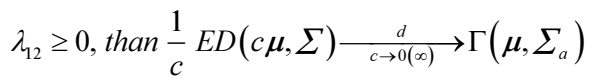

where, $\Gamma$ is the bivariate Gamma defined previously and

$$
\Sigma_{a}=\left[\begin{array}{cc}
\frac{1}{\alpha \lambda_{11}} & 0 \\
0 & \frac{1}{\alpha \lambda_{22}}
\end{array}\right]
$$

The theorem will be proved for $v$ regularly varying at zero ( $L$ slowly varying in infinity); the proof for $v$ regularly varying at infinity is similar.

Proof: Let $\boldsymbol{Z}=\left(Z_{1}, Z_{2}\right)^{T} \sim E D^{*}(\boldsymbol{\theta}, \boldsymbol{\Lambda})$ be the bivariate additive $E D M$ generated by $v$, constructed as described above with $M G F$ :

$$
M^{*}(\boldsymbol{s} ; \boldsymbol{\theta}, \boldsymbol{\Lambda})=\frac{\left[e^{\kappa\left(\theta_{1}+s_{1}, \theta_{2}\right)}\right]^{\lambda_{1}}\left[e^{\kappa\left(\theta_{1}+\theta_{2}, s_{2}\right)}\right]^{\lambda_{2}}\left[e^{\kappa\left(\theta_{1}+s_{1}, \theta_{2}+s_{2}\right)}\right]^{\lambda_{12}}}{\left[e^{\kappa\left(\theta_{1}+\theta_{2}\right)}\right]^{\lambda_{1}+\lambda_{2}+\lambda_{12}}} .
$$

Replacing by ( 5) we obtain:

$$
\begin{aligned}
& M^{*}(\boldsymbol{s} ; \boldsymbol{\theta}, \boldsymbol{\Lambda})=\left(1+\frac{s_{1}}{\theta_{1}}\right)^{-\alpha \lambda_{11}}\left(1+\frac{s_{2}}{\theta_{2}}\right)^{-\alpha \lambda_{22}} \\
& \times\left[\frac{L\left(-\theta_{1}-s_{1}, \theta_{2}\right)}{L\left(-\theta_{1}-, \theta_{2}\right)}\right]^{\lambda_{1}}\left[\frac{L\left(-\theta_{1},-\theta_{2}-s_{2}\right)}{L\left(-\theta_{1},-\theta_{2}\right)}\right]^{\lambda_{2}} \\
& \times\left[\frac{L\left(-\theta_{1}-s_{1},-\theta_{2}-s_{2}\right)}{L\left(-\theta_{1},-\theta_{2}\right)}\right]^{\lambda_{12}}
\end{aligned}
$$

and given the scale transformation $\boldsymbol{Y}=\left(Y_{1}, Y_{2}\right)^{T}=\left(\frac{Z_{1}}{\lambda_{11}}, \frac{Z_{2}}{\lambda_{22}}\right)^{T}$ and $M G F s$ properties, the $M G F$ for the perturbed reproductive model $\frac{1}{c} E D(c \mu, \Sigma)$ results, for $\mu_{i}>0$ fix, $i=1,2$ and $c$ small enough to ensure that $c \mu \in \Omega$ : 


$$
\begin{aligned}
M\left(\frac{1}{c} s ; \tau^{-1}(c \mu), \Lambda\right) & =\left(1+\frac{s_{1}}{\lambda_{11} c \delta_{1}(c \mu)}\right)^{-\alpha \lambda_{11}} \\
& \times\left(1+\frac{s_{2}}{\lambda_{22} c \delta_{2}(c \mu)}\right)^{-\alpha \lambda_{22}} \\
& \times\left[\frac{L\left(-\delta_{1}(c \mu)-\frac{s_{1}}{\lambda_{11} c}, \delta_{2}(c \mu)\right)}{L\left(-\delta_{1}(c \mu),-\delta_{2}(c \mu)\right)}\right]^{\lambda_{1}} \\
& \times\left[\frac{L\left(-\delta_{1}(c \mu),-\delta_{2}(c \mu)-\frac{s_{2}}{\lambda_{22} c}\right)}{L\left(-\delta_{1}(c \mu),-\delta_{2}(c \mu)\right)}\right]^{\lambda_{2}} \\
& \times\left[\frac{L\left(-\delta_{1}(c \mu)-\frac{s_{1}}{\lambda_{11} c},-\delta_{2}(c \mu)-\frac{s_{2}}{\lambda_{22} c}\right)}{L\left(-\delta_{1}(c \mu),-\delta_{2}(c \mu)\right)}\right]^{\lambda_{12}}
\end{aligned}
$$

Let us denote by $h_{i}(\boldsymbol{s} ; c, \boldsymbol{\mu}, \boldsymbol{\Lambda})$ the expressions with exponent $\lambda_{i}(i=1,2)$ and by $h_{12}(\boldsymbol{s} ; \boldsymbol{c}, \boldsymbol{\mu}, \boldsymbol{\Lambda})$ the one with exponent $\lambda_{12}$ so the $M G F$ can be written as follows:

$$
\begin{aligned}
M\left(\frac{1}{c} s ; \tau^{-1}(c \mu)\right. & , \Lambda)=\left(1+\frac{s_{1}}{\lambda_{11} c \delta_{1}(c \mu)}\right)^{-\alpha \lambda_{11}} \\
& \times\left(1+\frac{s_{2}}{\lambda_{22} c \delta_{2}(c \mu)}\right)^{-\alpha \lambda_{22}} \\
& \times h_{1}^{\lambda_{1}}(s ; c, \mu, \Lambda) \\
& \times h_{2}^{\lambda_{2}}(s ; c, \mu, \Lambda) \\
& \times h_{12}^{\lambda_{12}}(s ; c, \mu, \Lambda) .
\end{aligned}
$$

The following equalities will be proved:

$$
\begin{aligned}
& \lim _{c \rightarrow 0} c \delta_{i}(c \boldsymbol{\mu})=-\frac{\alpha}{\mu_{i}},=1,2, \\
& \lim _{c \rightarrow 0} h_{i}(s ; c, \mu, \Lambda)=1, i=1,2, \\
& \lim _{c \rightarrow 0} h_{12}(s ; c, \mu, \Lambda)=1 .
\end{aligned}
$$

To prove (9a) note that from (6) and given condition (7), we have that:

$$
\lim _{c \rightarrow 0} c \delta_{i}(c \boldsymbol{\mu})=\lim _{c \rightarrow 0} \frac{-\alpha}{\mu_{i}+\frac{1}{c} \frac{\partial l\left(-\tau^{-1}(c \boldsymbol{\mu})\right)}{\partial \delta_{i}(c \boldsymbol{\mu})}}=-\frac{\alpha}{\mu_{i}}
$$

Before proving (9b) and in order to simplify the notation we define $\tilde{\boldsymbol{\theta}}^{T}=\left(\tilde{\theta}_{1}, \tilde{\theta}_{2}\right)^{T}=\left(\delta_{1}(c \boldsymbol{\mu}), \delta_{2}(c \boldsymbol{\mu})\right)^{T}$ where $\delta_{i}(c \boldsymbol{\mu}), i$
$=1,2$ are strictly increasing functions, as is shown in Appendix B.

Now, to obtain $\lim _{c \rightarrow 0} h_{1}(s ; c, \mu, \Lambda)$, we apply (4), in such a way that $h_{1}(\boldsymbol{s} ; c, \boldsymbol{\mu}, \boldsymbol{\Lambda})$, with $\tau^{-1}(c \boldsymbol{\mu})$ in terms of $\tilde{\theta}$ can be expressed as:

$$
\begin{aligned}
& h_{1}(\boldsymbol{s} ; c, \tilde{\boldsymbol{\theta}}, \Lambda)=\frac{L\left(-\tilde{\theta}_{1}\left(1+z_{1}\right),-\tilde{\theta}_{2}\right)}{L\left(-\tilde{\theta}_{1},-\tilde{\theta}_{2}\right)} \\
& =\frac{d\left(-\tilde{\theta}_{1}\left(1+z_{1}\right),-\tilde{\theta}_{2}\right)}{d\left(-\tilde{\theta}_{1},-\tilde{\theta}_{2}\right)} \exp \left\{\int_{m}^{n} \frac{a(t, t)}{t} d t\right\}
\end{aligned}
$$

where, $\quad m=\left\|\left(-\tilde{\theta}_{1},-\tilde{\theta}_{2}\right)\right\|, n=\left\|\left(-\tilde{\theta}_{1}\left\{1+z_{1}\right\},-\tilde{\theta}_{2}\right)\right\|$, being $\|\cdot\|$ any norm and $z_{1}=\frac{s_{1}}{\lambda_{1} c \tilde{\theta}_{1}}$.

Taking into account that $\lim _{c \rightarrow 0}\left(1+z_{1}\right)=1-\frac{s_{1} \mu_{1}}{\lambda_{1} \alpha}>0$ because $s_{1}<0$ and given the conditions required for bivariate Karamata representation, it can be deduced that:

$$
\lim _{c \rightarrow 0} \frac{d\left(-\tilde{\theta}_{1}\left(1+z_{1}\right),-\tilde{\theta}_{2}\right)}{d\left(-\tilde{\theta}_{1},-\tilde{\theta}_{2}\right)}=\frac{d_{0}}{d_{0}}=1 .
$$

We also have that:

$$
a(t, t) \leq \sup _{m \leq t \leq n} a(t, t) \text { and that } \frac{1}{t} \leq \frac{1}{m},
$$

and then:

$$
\int_{m}^{n} \frac{a(t, t)}{t} d t \leq \sup _{m \leq t \leq n} a(t, t) \frac{1}{m}(n-m)
$$

For type 1 norm defined as $\|(x, y)\|_{1}=|x|+|y|$ we have that:

$$
\begin{aligned}
n-m & =\left|\tilde{\theta}_{1}\right|\left|1+z_{1}\right|+\left|\tilde{\theta}_{2}\right|-\left|\tilde{\theta}_{1}\right|-\left|\tilde{\theta}_{2}\right| \\
& =\left|\tilde{\theta}_{1}\right|\left(\left|1+z_{1}\right|-1\right)
\end{aligned}
$$

and:

$$
\int_{m}^{n} \frac{a(t, t)}{t} d t \leq \sup _{m \leq t \leq n} a(t, t) \frac{1}{\left|\tilde{\theta}_{1}\right|+\left|\tilde{\theta}_{2}\right|}\left|\tilde{\theta}_{1}\right|\left(\left|1+z_{1}\right|-1\right) .
$$

Now, given that $\frac{1}{\left|\tilde{\theta}_{1}\right|} \geq \frac{1}{\left|\tilde{\theta}_{1}\right|+\left|\tilde{\theta}_{2}\right|}$ :

$$
\lim _{c \rightarrow 0} \int_{m}^{n} \frac{a(t, t)}{t} d t \leq \lim _{c \rightarrow 0 m \leq t \leq n} a(t, t) \frac{1}{\left|\tilde{\theta}_{1}\right|}\left|\tilde{\theta}_{1}\right|\left(\left|1+z_{1}\right|-1\right)=0
$$

hence: 


$$
\lim _{c \rightarrow 0} \exp \left\{\int_{m}^{n} \frac{a(t, t)}{t} d t\right\}=1 \text {. }
$$

Putting together both results (11) and (12) and replacing in (10):

$$
\lim _{c \rightarrow 0} h_{1}(s ; c, \mu, \Lambda)=1 .
$$

Limits for $h_{2}$ and $h_{12}$ when $c \rightarrow 0$ can be obtained in a similar way, then taking limits in (8) we have that:

$$
\lim _{c \rightarrow 0} M\left\{\frac{1}{c} \boldsymbol{s} ; \tau^{-1}(c \boldsymbol{\mu}), \Lambda\right\}=\left(1-\mu_{1} \frac{s_{1}}{\alpha \lambda_{11}}\right)^{-\alpha \lambda_{11}}\left(1-\mu_{2} \frac{s_{2}}{\alpha \lambda_{22}}\right)^{-\alpha \lambda_{22}},
$$

and this is the expression for the $M G F$ of the bivariate dispersion model $\Gamma\left(\boldsymbol{\mu}, \Sigma_{\alpha}\right)$ for independent variables, as was proved in (2). The matrix $\Sigma_{\alpha}$ takes the following form:

$$
\Sigma_{a}=\left[\begin{array}{cc}
\frac{1}{\alpha \lambda_{11}} & 0 \\
0 & \frac{1}{\alpha \lambda_{22}}
\end{array}\right]
$$

We present next an example of a bivariate EDM generated by a regularly varying measure that satisfies the conditions required by Theorem 2 .

Example 1. Let the bivariate $E D M, E D(\boldsymbol{\mu}, \boldsymbol{\Sigma})$ be generated by the following measure, that is an extension of the measure presented by Letac (1992):

$$
v\left(d y_{1}, d y_{2}\right)=\left(e^{2 y_{1}}-1\right)\left(e^{2 y_{2}}-1\right) d y_{1} d y_{2},\left(y_{1}, y_{2}\right) \in \mathbb{R}_{+}^{2} .
$$

The $C G F$ is:

$$
\begin{aligned}
k\left(\theta_{1}, \theta_{2}\right) & =\log \int_{0}^{\infty} \int_{0}^{\infty} e^{y_{1} \theta_{1}+y_{2} \theta_{2}} \boldsymbol{v}\left(d y_{1}, d y_{2}\right) \\
& =\log \frac{2}{\theta_{1}^{2}+2 \theta_{1}}+\log \frac{2}{\theta_{2}^{2}+2 \theta_{2}} .
\end{aligned}
$$

In order to analyse if $v$ varies regularly we obtain the distribution function $\bar{v}\left(y_{1}, y_{2}\right)=\boldsymbol{v}\left\{\left(0, y_{1}\right] \times\left(0, y_{2}\right]\right\}$.

$$
\begin{aligned}
\bar{v}\left(y_{1}, y_{2}\right) & =\int_{0}^{y_{1}}\left(e^{2 u}-1\right) d u \int_{0}^{y_{2}}\left(e^{2 s}-1\right) d s \\
& =\left[\frac{e^{2 y_{1}}}{2}-y_{1}-\frac{1}{2}\right]\left[\frac{e^{2 y_{2}}}{2}-y_{2}-\frac{1}{2}\right]
\end{aligned}
$$

and taking limits:

$$
\begin{aligned}
\lim _{t \rightarrow 0} \frac{\bar{v}\left(t y_{1}, t y_{2}\right)}{\bar{v}(t, t)} & =\lim _{t \rightarrow 0} \frac{e^{2 t y_{1}}-2 t y_{1}-1}{e^{2 t}-2 t-1} \frac{e^{2 t y_{2}}-2 t y_{2}-1}{e^{2 t}-2 t-1} \\
& =\left(y_{1} y_{2}\right)^{2},
\end{aligned}
$$

then $\bar{v} \in V R(2,2)_{0}$ and by Definition 2 the measure $v$ varies regularly.

Given (3) we can affirm that the $M G F$ is:

$$
\begin{aligned}
M_{v}\left(\theta_{1}, \theta_{2}\right) & =e^{\kappa(\theta)} \\
& =\frac{2}{\theta_{1}^{2}+2 \theta_{1}} \frac{2}{\theta_{2}^{2}+2 \theta_{2}} \\
& =\left(-\theta_{1}\right)^{-2}\left(-\theta_{2}\right)^{-2} L\left(-\theta_{1},-\theta_{2}\right),
\end{aligned}
$$

where, $L \in V L_{\infty}$ is:

$$
L\left(-\theta_{1},-\theta_{2}\right)=\frac{4 \theta_{1} \theta_{2}}{\left(\theta_{1}+2\right)\left(\theta_{2}+2\right)} .
$$

Let us analyse conditions (7):

$$
\frac{\partial l\left(-\theta_{1},-\theta_{2}\right)}{\partial \theta_{i}}=\frac{1}{\theta_{i}}-\frac{1}{\theta_{i}+2}=\frac{2}{\theta_{i}\left(\theta_{i}+2\right)} i=1,2
$$

and taking into account that:

$$
\frac{\partial^{2} k}{\partial \theta_{i} \partial \theta_{i}}=\frac{2}{\theta_{i}^{2}+2 \theta_{i}}+\frac{4\left(\theta_{i}+1\right)^{2}}{\left(\theta_{i}+2\right)^{2}} i=1,2,
$$

these second derivatives can be expressed in terms of mean values:

$$
\ddot{\kappa}_{\theta_{i} \theta_{i}}\left(\theta_{1}, \theta_{2}\right)=V_{i}\left(\mu_{i}\right)=\mu_{i}^{2}+1-\sqrt{\mu_{i}^{2}+1} i=1,2,
$$

giving:

$$
\frac{\partial l\left(-\theta_{1},-\theta_{2}\right)}{\partial \theta_{i}} \sqrt{\mu_{i}^{2}+1}-1 i=1,2 .
$$

Then, taking limits and applying L' Hopital:

$$
\begin{aligned}
\lim _{c \rightarrow 0} \frac{1}{c} \frac{\partial l\left(-\tilde{\theta}_{1},-\tilde{\theta}_{2}\right)}{\partial \tilde{\theta}_{i}} & =\lim _{c \rightarrow 0} \frac{\sqrt{c^{2} \mu_{i}^{2}+1-1}}{c} \\
& =\lim _{c \rightarrow 0} \frac{1}{2}\left(c^{2} \mu_{i}^{2}+1\right)^{-\frac{1}{2}} 2 c \mu_{i}^{2}=0,
\end{aligned}
$$

for $\mathrm{i}=1,2$, so conditions (7) are satisfied. Now, given (4) and according with Theorem 2, EDMs generated by (13) satisfy that when $c \rightarrow 0$ :

$$
\frac{1}{c} E D\left(c \mu, \Sigma_{a}\right) \stackrel{d}{\longrightarrow} \Gamma\left(\mu, \Sigma_{a}\right)
$$

$\Gamma$ being the bivariate Gamma distribution for independent variables with $\Sigma_{a}=\left[\begin{array}{cc}\frac{1}{2 \lambda_{11}} & 0 \\ 0 & \frac{1}{2 \lambda_{22}}\end{array}\right]$. 


\section{Conclusion and Discussion}

The Gaussian distribution has been for a long time the main tool of most statistical analysis, although Fisher (1953) pointed out the importance of describing data in their natural habitat. Gaussian distribution is particularly relevant for small dispersion data, but it does not include the case of large dispersion. Jørgensen developed a theory for large dispersion models that covers infinite distributions that can be chosen as the most appropriate for each data set.

While dealing with multivariate responses in the nonormal case it is necessary to extend classical theory to flexible multivariate distribution families. Statistical literature provides a large variety of such families, but it is not easy to choose between them as pointed out by Letac (2007). With these considerations in mind, the search was reasonably based in the identification of multivariate distributions that keep some of the characteristics of the corresponding univariate distribution, being marginal and conditionally closed. This is not an easy task, Jørgensen (2013; Jørgensen and Martínez, 2013) presented a new class of MEDMs with the ideal number of parameters; they based the construction on an extension of the convolution method.

On the other hand, studies about convergence of EDMs performed by Jørgensen et al. (1994) and Jørgensen et al. (2009) allowed these authors to conjecture that similar results could be proved for those MEDMs they defined in 2013. Contributions made by $\mathrm{J}$. Karamata are relevant for this type of convergence, he extended to $\mathbb{R}^{k}$ some Tauberian theorems for regular variation functions establishing that those functions behave asymptotically as their Laplace transforms.

In this article we proved that bivariate EDMs generated by regularly varying measures, tend to a bivariate independent Gamma distribution when the mean parameter goes to some extreme in the parameter domain. This extension of Tauber type convergence to a Gamma model is done imposing no conditions on the asymptotic behaviour of the variance function in a similar way as the univariate theorem.

Only measures of regular variation type with the same index for both variables have been considered, because models generated by measures with different index for each variable would imply the existence of bivariate models whose variance function has is different for each variable, this situation is hard to imagine.

Recently, Hitz and Evans (2016) developed an extension of Karamata theorem to multivariate regular variation functions and their results are important in extreme value theory opening a new line of research of convergence properties of dispersion models for extremes, taking into account the parallelism between those models and EDMs developed in (Jørgensen et al., 2010).

\section{Acknowledgement}

This research has been supported by grant EXAM/18 from the Universidad Nacional de Mar del Plata and by grant CS 4594/17 from the Universidad Nacional de Rosario.

\section{Author's Contributions}

Each author contributed with a proportion of $50 \%$ in the preparation, development and publication of this manuscript.

\section{Ethics}

This article is original and contains unpublished material. The corresponding author confirms that all of the other authors have read and approved the manuscript and no ethical issues involved.

\section{Appendixes}

\section{A. Proof of Proposition 1}

We will demonstrate the following equivalence:

$$
\bar{v}(t, s) \sim \frac{1}{\Gamma(\alpha+1) \Gamma(\beta+1)} t^{\alpha} s^{\beta} L(t, s) \text { when }
$$

$\min (t, s) \rightarrow \infty \Leftrightarrow \bar{v} \in V R(\alpha, \beta)_{\infty}$ where $\alpha, \beta \in \mathbb{R}^{+}$.

Proof. When $\min (t, s) \rightarrow \infty$ we can write:

$$
L(t, s) \sim \frac{\Gamma(\alpha+1) \Gamma(\beta+1) \bar{v}(t, s)}{t^{\alpha} s^{\beta}} .
$$

Also, given that $L \in V L_{\infty}$,

$$
\lim _{\min (a, b) \rightarrow \infty} \frac{L(a t, b s)}{L(a, b)}=1 .
$$

Replacing by (14), we obtain:

$$
\lim _{\min (a, b) \rightarrow \infty} \frac{\bar{v}(a t, b s)}{\bar{v}(a, b)}=t^{\alpha} s^{\beta}
$$

meaning that $\bar{v}(t, s) \in V R(\alpha, \beta)_{\infty}$.

\section{B. Properties of $\tilde{\theta}_{i}, i=1,2$}

To prove that $\tilde{\theta}_{i}, i=1,2$ are strictly increasing functions, we will compute their derivatives:

$$
\frac{d}{d c} \delta_{i}\left(c \mu_{1}, c \mu_{2}\right)=\frac{\partial}{\partial\left(c \mu_{1}\right)} \delta_{i}(c \boldsymbol{\mu}) \mu_{1}+\frac{\partial}{\partial\left(c \mu_{2}\right)} \delta_{i}(c \boldsymbol{\mu}) \mu_{2} .
$$


The Jacobian matrix is:

$$
J\left[\begin{array}{l}
\delta_{1}(c \boldsymbol{\mu}) \\
\delta_{2}(c \boldsymbol{\mu})
\end{array}\right]=\left[\begin{array}{ll}
\frac{\partial \delta_{1}(c \boldsymbol{\mu})}{\partial\left(c \mu_{1}\right)} & \frac{\partial \delta_{1}(c \boldsymbol{\mu})}{\partial\left(c \mu_{2}\right)} \\
\frac{\partial \delta_{2}(c \boldsymbol{\mu})}{\partial\left(c \mu_{1}\right)} & \frac{\partial \delta_{2}(c \boldsymbol{\mu})}{\partial\left(c \mu_{2}\right)}
\end{array}\right]=J \tau^{-1}(c \boldsymbol{\mu}),
$$

and it is known that $J \tau^{-1}(c \boldsymbol{\mu})=(J \tau(\tilde{\theta}))^{-1}$, then:

$$
\begin{aligned}
& J \tau^{-1}(c \mu)=\left[\begin{array}{ll}
V_{1}\left(c \mu_{1}\right) & V_{12}(c \mu) \\
V_{12}(c \mu) & V_{2}\left(c \mu_{2}\right)
\end{array}\right]^{-1} \\
& =\frac{1}{\Delta}\left[\begin{array}{cc}
V_{2}\left(c \mu_{2}\right) & -V_{12}(c \mu) \\
-V_{12}(c \mu) & V_{1}\left(c \mu_{1}\right)
\end{array}\right]
\end{aligned}
$$

where, $\Delta=V_{1}\left(c \mu_{1}\right) V_{2}\left(c \mu_{2}\right)-V_{12}^{2}(c \mu)$.

Then:

$$
\frac{d}{d c} \delta_{1}(c \mu)=\frac{1}{\Delta}\left(V_{2}\left(c \mu_{2}\right) \mu_{1}-V_{12}(c \mu) \mu_{2}\right)
$$

and:

$$
\frac{d}{d c} \delta_{2}(c \boldsymbol{\mu})=\frac{1}{\Delta}\left(V_{1}\left(c \mu_{1}\right) \mu_{2}-V_{12}(c \mu) \mu_{2}\right)
$$

Both expressions are always positive. Then we have that $\delta_{i}(c \boldsymbol{\mu})$ are strictly increasing functions. Then $c \rightarrow 0$ implies $\tilde{\theta}_{1} \rightarrow-\infty$ and $\tilde{\theta}_{2} \rightarrow-\infty$ for $\mu_{1}$ and $\mu_{2}$ fixed.

\section{Reparametrization of Kibble and Moran bivariate Gamma Variance Function}

The mean and the variance function corresponding to Kibble and Moran bivariate gamma distribution are given by the gradient and the Hessian of $\kappa(\boldsymbol{\theta})$ :

$$
\mu=\dot{\kappa}(\theta)=\frac{1}{\rho-\theta_{1} \theta_{2}}\left[\begin{array}{c}
\theta_{2} \\
\theta_{1}
\end{array}\right],
$$

giving $\mu_{1}=\frac{\theta_{2}}{\rho-\theta_{1} \theta_{2}}$ and $\mu_{2}=\frac{\theta_{1}}{\rho-\theta_{1} \theta_{2}}$. Also:

$$
V(\boldsymbol{\mu})=\ddot{\kappa}(\theta)=\frac{1}{\left(\rho-\theta_{1} \theta_{2}\right)^{2}}\left[\begin{array}{cc}
\theta_{2}^{2} & \rho \\
\rho & \theta_{1}^{2}
\end{array}\right]
$$

gives the correlation coefficient $\phi=\frac{\rho}{\theta_{1} \theta_{2}}$.

From (16) we can re parametrize the diagonal elements in (17) as $[V(\mu)]_{i i}=\mu_{i}^{2}, i=1,2$. The elements off the diagonal are:

$$
[V(\boldsymbol{\mu})]_{i j}=\frac{\rho}{\left(\rho-\theta_{1} \theta_{2}\right)^{2}}=\mu_{1} \mu_{2} \frac{\rho}{\theta_{1} \theta_{2}}=\mu_{1} \mu_{2} \phi
$$

Now the variance function can be re-written in terms of mean value parameters:

$$
V(\boldsymbol{\mu})=\left[\begin{array}{cc}
\mu_{1}^{2} & \phi \mu_{1} \mu_{2} \\
\phi \mu_{1} \mu_{2} & \mu_{1}^{2}
\end{array}\right] .
$$

We need to express $\phi$ in terms of $\mu_{1}, \mu_{2}$ and $\rho$. We first clear up $\theta_{1}$ and $\theta_{2}$ :

$$
\begin{aligned}
& \mu_{1}=\frac{\theta_{2}}{\rho-\theta_{1} \theta_{2}}=\frac{1}{(\phi-1) \theta_{1}} \Rightarrow \theta_{1}=\frac{1}{(\phi-1) \mu_{1}} \\
& \mu_{2}=\frac{\theta_{1}}{\rho-\theta_{1} \theta_{2}}=\frac{1}{(\phi-1) \theta_{2}} \Rightarrow \theta_{2}=\frac{1}{(\phi-1) \mu_{2}}
\end{aligned}
$$

giving:

$$
\phi=\frac{\rho}{\theta_{1} \theta_{2}}=\rho \mu_{1} \mu_{2} \phi^{2}-2 \rho \mu_{1} \mu_{2} \phi+\rho \mu_{1} \mu_{2}
$$

and after some algebraic manipulations, we obtain the second order equation:

$$
\rho \mu_{1} \mu_{2} \varphi^{2}-\left(\rho \mu_{1} \mu_{2}+1\right) \phi+\rho \mu_{1} \mu_{2}=0 .
$$

We choose the solution that gives a correlation coefficient $<1$ :

$$
\phi=1-\frac{1}{2 \rho \mu_{1} \mu_{2}}\left(\sqrt{4 \rho \mu_{1} \mu_{2}+1}-1\right) .
$$

\section{References}

Boggio, G., 2019. Teoremas de convergencia para modelos exponenciales con dispersion bivariados. $\mathrm{PhD}$ Thesis, Universidad Nacional de Rosario, Rosario, Argentina.

de Haan, L. and S. Resnick, 1987. On regular variation of probability densities. Stochastic Processes Applic., 25: 83-93.

de Haan, L., 1975. On regular variation and its applications to the weak convergence of sample extremes. Math. Centre Tracts. Centrum, Amsterdam.

Fisher, R.A., 1953. Dispersion on a sphere. Royal Society Series A, 217: 295-305.

Goldie, C.M., N.H. Bingham and J.L. Teugels, 1987. Regular Variation. 1st Edn., Cambridge University Press, Cambridge. 
Hitz, A. and R. Evans, 2016. One-component regular variation and graphical modeling of extremes. J. Applied Probab., 53: 733-746.

Cuenin, J., B. Jørgensen and C. Kokonendji, 2015. Simulations of full multivariate Tweedie with flexible dependence structure. Comput. Stat., 31: 09-09.

Jørgensen, B. and J.R. Martínez, 2013. Multivariate exponential dispersion models. Multivariate Stat. Theory Applied.

Jørgensen, B., 1987. Exponential dispersion models (with discussion). J. Royal Stat. Society Series B, 49: 127-162.

Jørgensen, B., 1997. The theory of dispersion models. Chapman Hall.

Jørgensen, B., 2013. Construction of multivariate dispersion models. Brazilian J. Probability Stat., 27: 285-309.

Jørgensen, B., J.R. Martınez and M. Tsao, 1994. Asymptotic behavior of the variance function. Scandinavian J. Stat., 21: 223-243.

Jørgensen, B., J.R. Martínez and V. Vinogradov, 2009. Domains of attractions to Tweedie distributions. Lithuanian Math. J., 49: 399-425.
Jørgensen, B., Y. Goegebeur and J.R. Martínez, 2010. Dispersion models for extremes. Extremes, 13: 399-437.

Kotz, S., N. Balakrishnan and N.L. Johnson, 2000. Continuous Multivariate Distributions. 2nd Edn., John Wiley and Sons.

Letac, G., 2007. The bivariate gamma distribution with Laplace transform $(1+a s+b t+c s t)^{-q}$ : History, characterizations, estimation.

Nelder, J.A. and R.W.M. Wedderburn, 1972. Generalized linear models. J. Royal Stat. Society. Series A, 135: 370-384.

Nielsen, R., 2000. Exponential dispersion models and Tweedie convergence. MSc Thesis, University of Southern Denmark.

Omey, E. and E. Willekens, 1989. Abelian and Tauberian theorems for the Laplace transform of functions in several variables. J. Multivariate Analysis, 30: 292-306.

Tweedie, M.C.K., 1947. Functions of statistical variate with given means, with special reference to Laplacian distributions. Cambridge Philosophical Society, 49: 41-49. 